\title{
The changing risk of vector-borne diseases: Global satellite remote sensing and geospatial surveillance at the forefront
}

\author{
Robert Bergquist, ${ }^{1}$ Jeffrey C. Luvall, ${ }^{2}$ John B. Malone ${ }^{3}$ \\ ${ }^{1}$ Ingerod, Brastad, Sweden; ${ }^{2}$ National Aeronautics Space Administration (NASA), Huntsville, AL, USA; \\ ${ }^{3}$ Department of Pathobiological Sciences, Louisiana State University, Baton Rouge, LA, USA
}

\section{Introduction}

The threat of climate change has already translated into early signs of a changing distribution of vector-borne diseases; where will they go next? The global, average temperature, regularly recorded since 1880 , has passed $1^{\circ} \mathrm{C}$ above the mean of the period monitored. In addition, the higher the latitude, the more pronounced the temperature change.

On 6 August 2021, the United Nations Intergovernmental Panel on Climate Change (IPCC) released the report by its working group on the Physical Basis of Climate Change (IPCC, 2021). There are two other working groups: one on Impacts, Adaptation and Vulnerability, the other on Mitigation of Climate Change; together with the first group they will jointly issue IPCC's Sixth Assessment Report (AR6) next year. The already available working group report is the most elaborate attempt so far to understand the climate system and it warns that an average global warming by $1.5^{\circ} \mathrm{C}$ can only be avoided if massive and immediate cuts in greenhouse gas emissions are made. Thus, we are dangerously near a tipping point that could lead to irreversible climate changes.

\section{Supporting observations}

The broad spectrum of climate data from the instruments onboard today's Earth-observing satellites have facilitated mapping and statistical analysis of the distribution of vector-borne diseases. Although this technology is advanced, the thinking it is based on is far from new. As early as in the beginning of the $18^{\text {th }}$ century, René Antoine de Réaumur came up with the degree-day, a merged unit of temperature and time describing the relationship between plant development and the ambient temperature (Bonhomme, 2000). This unit, defined as the amount of heat an

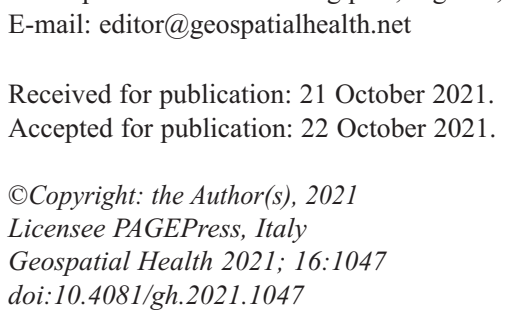

This article is distributed under the terms of the Creative Commons Attribution Noncommercial License (CC BY-NC 4.0) which permits any noncommercial use, distribution, and reproduction in any medium, provided the original author(s) and source are credited. organism must accumulate to reach full development, is now referred to as the growing-degree-day (GDD) and used for widely different measurements, including the development of parasites and their vectors (Bernal, 1993). With respect to vectors, the GDD approach constituted a major step forward (Valencia-López et al., 2012; Wang et al., 2021, Potential risk of colonization of Bulinus globosus and Schistosoma haematobium in Mainland China, unpublished) by focusing on Nature as a set of contiguous niches with diverse ecology. This view was first outlined by Pavlovskii (1945) and later developed by Nix (1986), who developed a habitat-model showing which niche would be suitable for a species under past, present or future conditions. Current research in this area is supported by sources such as the WorldClim database (Hijmans et al., 2005; Fick and Hijmans, 2017), that contains variables derived from remotely sensed information of temperatures and rainfall interpolated with data from terrestrial weather stations. The results have contributed to the recent finding that many vector-borne diseases are moving northward, both in tropical, endemic areas (Khormi and Kumar, 2016; Stensgaard et al., 2019) and in temperate zones (Zhou et al., 2008; Asghar et al., 2016; Wang et al., 2021, Potential risk of colonization of Bulinus globosus and Schistosoma haematobium in Mainland China, unpublished).

For those interested in the evolutionary study of ancient infections, there is now also PaleoClim (Brown et al., 2018), a free database (http://www.paleoclim.org) of past climates at a $5-\mathrm{km}^{2}$ resolution that includes simulations based on a climate circulation model based on prehistoric data made available by the Met Office Hadley Centre in the UK. So far, this database contains climatic data for some key time periods spanning from 3.3 to 0.8 million years ago (Ma): the Marine Isotope Stage 19 (MIS19) in the Pleistocene $(\sim 0.79 \mathrm{Ma})$ and the middle to late Pliocene warm period ( 3.26-3.03 Ma). The availability of climatic drill core data indicating the conditions of previous climates, e.g., that of the late Pleistocene and Holocene have revolutionized the study of species in those early days. Recent excavations of ancient areas have revealed the incidence of human and animal parasitic infections in the Middle East dating back to 8100 BC (Khodkar et al., 2018), while Banks (2017) used eco-cultural niche modelling as a way to understand the dynamics of European culture changes as far back as 40,000 years ago.

\section{Remote sensing}

Remote sensing can be used to measure, evaluate or estimate both the environment (state functions) using thermal remote sensing (Luvall et al., 1990; Quattrochi and Luvall, 2014) and interfaces (process functions) using hyper-spectral remote sensing (Asner et al., 2009), e.g., for defining vector habitats. The remotely sensed products can be integrated directly into the epidemiolog- 
ical equations to significantly enhance our understanding of habitats and life cycles of vectors and also those of the parasites they harbour. Remotely sensed information can today specify niche characteristics in great detail allowing collection of information at the household level (Malone et al., 2019).

As novel instruments for earth observation and near real time surveillance applications emerge, they may initially suffer from lack of a previous time series for essential temporal context. Landsat 9, launched from Vandenberg Space Force Base on September 27, 2021, takes the place of Landsat 7, a satellite that orbited 8 days out of phase with Landsat 8 , thus continuing the Landsat legacy dataset that has so far provided compatible imagery since 1972, a stable standard to compare anticipated value of new Earth observing systems. Other long-term time series data are provided by MODIS (the Moderate Resolution Imaging Spectroradiometer) and the more elaborate VIIRS (the Visible Infrared Imaging Radiometer Suite) that succeeded it. For almost 20 years, MODIS has been viewing the entire Earth every 1 to 2 days in 36 different spectral bands and 3 different spatial resolutions, deriving sea surface temperature, land surface temperature, vegetation health and aerosol concentrations. These coordinated systems based onboard polar-orbiting satellites allow us to view our planet holistically, considering a broad range of environmental variables with full global coverage twice a day.

The current instruments on the International Space Station (ISS), launched by NASA (the National Aeronautics and Space Administration), are providing 'pathfinder' datasets for the design of the next generation global satellite observations. The ISS instrumentation includes three important, health-related sensors: ECOSTRESS (the ECOsystem Spaceborne Thermal Radiometer Experiment on Space Station) that provides global data focused on agriculture and vegetation via 5-channel, thermal infrared (IR) measurements with 70-m resolution and a 2-5 day repeat cycle of day/night pairs; DESIS [the German Aerospace Center (DLR) Earth Sensing Imaging Spectrometer] that depicts land surface, oceans and the atmosphere with unparalleled accuracy from its hyper-spectral sensor system with 235 channels and 30-m resolution, jointly developed by the DLR and Teledyne Brown Engineering in the United States; and GEDI (the Global Entrepreneurship and Development Index) that observes Earth's forest structure and topography through a high-resolution laser ranger that provides global coverage. However, the most exciting NASA programme that will provide future global datasets significant to the public health community is the Earth System Observatory (ESO).

\section{Earth System Observatory and planned satellite launches}

On 5 January 2018, the Committee on the Decadal Survey for Earth Science and Applications from Space (ESAS) of the National Academies of Sciences, Engineering and Medicine (NASEM) Space Studies Board, Division on Engineering and Physical Sciences released the 2017 Decadal Survey that can be downloaded at https://www.nap.edu/catalog/25437/thriving-onour-changing-planet-a-decadal-strategy-for-earth-observationfrom-space and in June 2021 the ESO was announced. The Decadal Survey addresses 35 key science and applications questions, with those prioritized falling into the following six categories: Coupling of water and energy cycles; Ecosystem change; Extending and improving weather and air quality forecasts; Reducing climate uncertainty and informing societal response; Sea level rise; and Surface dynamics, geological hazards and disasters. The ESO will be accomplished by five designated, foundational satellite missions planned for the coming decade:

- Aerosols: Answering the critical question of how aerosols affect the global energy balance, a key source of uncertainty in predicting climate change;

- Cloud, convection and precipitation: Tackling the largest sources of uncertainty in future projections of climate change, air quality forecasting and prediction of severe weather;

- Mass change: Providing drought assessment and forecasting, associated planning for water use for agriculture as well as supporting natural hazard response;

- Surface biology and geology: Understanding climate changes that impact food and agriculture, habitation and natural resources, by answering open questions about the fluxes of carbon, water, nutrients and energy within and between ecosystems and the atmosphere, the ocean and the Earth; and

- Surface deformation and change: Quantifying models of sealevel and landscape change driven by climate change, hazard forecasts and disaster impact assessments, including dynamics of earthquakes, volcanoes, landslides, glaciers, groundwater and Earth's interior.

The first scheduled to launch and most important component of ESO for the public health community is the Surface Biology and Geology (SBG) mission, now in early stages of formulation for launch in the 2026-27 timeframe (Thomson et al., 2020). SBG will provide global measurements from two different satellites. First, a visible-to-shortwave, infrared (VSWIR) imaging spectroscopy instrument with $\sim 254$ channels from 0.35 to 2.5 micron with a 10 $\mathrm{nm}$ bandwidth at $30-\mathrm{m}$ resolution and a 16 day revisit. Second, a multispectral, thermal infrared (TIR) instrument with $>5$ channels in the 8 to 12 micron and a 3-5 micron range at 50-m resolution and 2-5 day visit with corresponding day-night pairs. In contrast to DESIS, whose data acquisitions are limited to areas ordered, the SBG hyperspectral instrument will have continuous global coverage.

In addition, the European Space Agency (ESA) is also starting the development of similar satellite missions, such as the Copernicus Hyperspectral Imaging Mission (CHIME) that includes VSWIR and the Land Surface Temperature Monitoring (LSTM) with the TIR payload. There are also the current Italian 'PRecursore IperSpettrale della Missione Applicativa' (PRISMA), a polarized radiation imaging and spectroscopy mission based on VSWIR, and the French-Indian Thermal infraRed Imaging Satellite for High resolution Natural resource Assessment (Trishnia) equipped with TIR.

\section{Points to ponder}

Climate-models can predict which factors would be of interest for disease control activities but forecasting suffers from ambiguity even concerning the immediate future, say the next decades. As demonstrated in drill core studies of 11,700 years old layers (Bond et al., 2001), even minor changes in solar output can lead to comparatively strong climate variations on Earth, e.g., those at the end of the last glacial period. The Sun's activity has recently shown a declining tendency, raising concerns that we might again be heading for a minimum, perhaps not as strong as the years recorded by Bond (2001), but it could possibly emulate the Maunder minimum in the late 1600 s that ushered in a cold-spell that lasted for 60 years (Maunder, 1894). Indeed, the Sun has shown a lengthening of its 11 -year cycles since the 1990s, and we are now entering a critically important cycle that could be a harbinger of the future solar activ- 
ity (Miyahara et al., 2021). The Sun's energy balance is much too complicated to describe here, but it might be useful to observe the energy exchange between Sun and Earth since even short-term changes would contribute to a better understanding. It might even provide a breathing space in the fight against global warming.

\section{References}

Asghar N, Petersson M, Johansson M, Dinnetz P, 2016. Local landscape effects on population dynamics of Ixodes ricinus. Geospat Health 11:487.

Asner GP, Martin RE, Ford AJ, Metcalfe DJ, Liddell MJ, 2009. Leaf Chemical and Spectral Diversity in Australian Tropical Forests. Ecol Appl 19:236-53.

Banks WE, 2017.The application of ecological niche modeling methods to archaeological data in order to examine culture environment relationships and cultural trajectories. Quaterinaire 28:271-6.

Bernal J, Gonzalez D, 1993. Experimental assessment of a degreeday model for predicting the development of parasites in the field. J Appl Ent 116:459-66.

Bond G, Kromer B, Beer J, Muscheler R, Evans MN, Showers W, Hoffmann S, Lotti-Bond R, Hajdas I, Bonani G, 2001. Persistent solar influence on North Atlantic climate during the Holocene. Science 294:2130-6.

Bonhomme R, 2000. Bases and limits to using 'degree.day' units. Eur J Agron 13:1-10.

Brown JL, Hill DJ, Dolan AM, Carnaval AC, Haywood AM, 2018. PaleoClim, high spatial resolution paleoclimate surfaces for global land areas. Sci Data 5:180254.

Fick SE, Hijmans RJ, 2017. Worldclim 2: New 1-km spatial resolution climate surfaces for global land areas. Int J Climatol 37:4302-15.

Hijmans RJ, Cameron SE, Parra JL, Jones PG, Jarvis A, 2005. Very high resolution interpolated climate surfaces for global land areas. Available from the WorldClim data website: http://www.worldclim.org/version1

IPCC, 2021. In: Masson-Delmotte V, Zhai P, Pirani A, Connors SL, Péan C, (Eds.), Climate Change 2021: The physical science basis. Contribution of Working Group I to the Sixth Assessment Report of the Intergovernmental Panel on Climate Change. Cambridge University Press, Cambridge, UK.

Khormi HM, Kumar L, 2016. Future malaria spatial pattern based on the potential global warming impact in South and Southeast Asia.
Geospat Health 11:416.

Khodkar I, Feizhadad MH, Tavalla M, 2018. Paleoparasitology in Iran: a review. Infez Med 26:396-402.

Luvall JC, Lieberman D, Lieberman M, Hartshorn GS, Peralta R, 1990. Estimation of tropical forest canopy temperatures, thermal response numbers, and evapotranspiration using an aircraft-based thermal sensor. Photogramm Eng Remote Sensing 56:1393-401.

Miyahara H, Tokanai F, Moriya T, Takeyama M, Sakurai H, Horiuchi K, Hotta H, 2021. Gradual onset of the Maunder Minimum revealed by high-precision carbon-14 analyses. Sci Rep 11:5482.

Maunder EW, 1894. A prolonged sunspot minimum. Knowledge 17:173-6.

Nix HA, 1986. A biogeographic analysis of Australian ela-pidsnakes. In: Longmore R (Ed.), Atlas of elapid snakes of Australia: Australian Flora and Fauna series no. 7. Bureau of Flora and Fauna, Canberra, Australia, pp. 4-15.

Pavlovskii EN, 1945. The ecological parasitology. J Gen Biol 6:65-92.

Malone JB, Bergquist R, Martins M, Luvall JC, 2019. Use of geospatial surveillance and response systems for vector-borne diseases in the elimination phase. Trop Med Infect Dis 4:pii:15.

Quattrochi DA, Luvall JC, 2014. Thermal infrared remote sensing for analysis of landscape ecological processes: current insights and trends; Chapter 3. In: Weng Q (Ed.), Scale issues in remote sensing. Wiley Blackwell, Hoboken, NJ, USA, pp. 34-60.

Stensgaard AS, Vounatsou P, Sengupta ME, Utzinger J, 2019. Schistosomes, snails and climate change: Current trends and future expectations. Acta Trop 190:257-68.

Thompson DR, Schimel DS, Poulter B, Brosnan I, Hook SJ, Green RO, Glenn N, Guild L, Henn C, Cawse-Nicholson K, Kokaly R, Lee C, Luvall J, Miller CE, Nastal CE, Pavlick R, Philips B, Schneider F, Schollaert Uz S, 2020. NASA's surface biology and geology concept study: status and next steps. pp. 32693271 in IGARSS 2020-2020 IEEE International Geoscience and Remote Sensing Symposium.

Valencia-López N, Malone JB, Carmona CG, Velásquez LE, 2012. Climate-based risk models for Fasciola hepatica in Colombia. Geospat Health 6:S67-85.

Zhou XN, Yang GJ, Yang K, Wang XH, Hong QB, Sun LP, Malone JB, Kristensen TK, Bergquist NR, Utzinger J, 2008. Potential impact of climate change on schistosomiasis transmission in China. Am J Trop Med Hyg 78:188-94. 